\title{
Persistence of Residues after Endodontic Retreatment related to the Obturation Technique and to the Solvent
}

\author{
${ }^{1}$ Arturo J Aranda-Garcia, ${ }^{2}$ Milton C Kuga, ${ }^{3}$ Fernando A Vázquez-Garcia, ${ }^{4}$ Mateus R Tonetto, ${ }^{5}$ Matheus C Bandeca \\ ${ }^{6}$ Suellen NL Lima, ${ }^{7}$ Joana Freitas, ${ }^{8}$ Alvaro H Borges, ${ }^{9}$ Keren CF Jordão-Basso, ${ }^{10}$ Jardel C do Carmo Monteiro
}

\begin{abstract}
Objective: To quantify the persistence of residues after endodontic retreatment related to the root canal obturation technique (lateral compaction or single cone) previously performed and the solvent used (xylol or Endosolv-R) in the cervical and root apical segment using digital radiograph.
\end{abstract}

Materials and methods: Forty root canals were prepared using the $\mathrm{F} 2$ instrument and filled with gutta-percha and $\mathrm{AH}$ Plus, 20 canals using \#25 point by lateral compaction and the other 20 using F2 point by single cone. The root canals were transversely sectioned, and two segments (cervical and apical) were obtained. Radiographs were taken from the segment in buccolingual and mesiodistal direction, as well as the area that was originally filled with the root canal obturation was measured using ImageJ. After the specimens were set on special devices, the obturations were removed using the rotary system and divided into four groups $(\mathrm{n}=10)$ : $\mathrm{G} 1$ - lateral compaction and xylol; G2 - lateral compaction and Endosolv-R; G3 - single cone and xylol; and G4 - single cone and Endosolv-R. A new radiograph was taken and the area occupied by residues was measured. The persistence of residues was obtained from the obturation remaining in the root canal in percentage. The data were analyzed using Kruskal-Wallis test $(p=0.05)$.

Results: There was no difference among the examined groups, regardless of the radicular segment or the radiograph direction $(p>0.05)$.

Conclusion: The endodontic retreatment using xylol or Endosolv- $\mathrm{R}$ in the obturations previously performed by lateral compaction or single cone technique presented similar persistence of residues in the root canal, regardless of the radicular segment.

Keywords: Endodontic, Retreatment, Root canal, Rotary Instruments, Solvents.

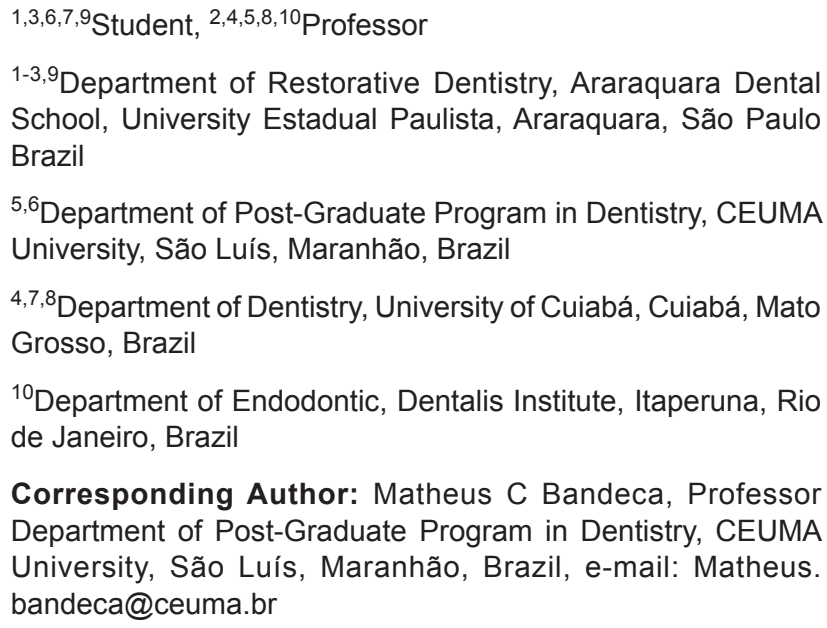

Corresponding Author: Matheus C Bandeca, Professor Department of Post-Graduate Program in Dentistry, CEUMA University, São Luís, Maranhão, Brazil, e-mail: Matheus. bandeca@ceuma.br

How to cite this article: Aranda-Garcia AJ, Kuga MC, Vázquez-Garcia FA, Tonetto MR, Bandeca MC, Lima SNL, Freitas J, Borges AH, Jordão-Basso KCF, do Carmo Monteiro JC. Persistence of Residues after Endodontic Retreatment related to the Obturation Technique and to the Solvent. World J Dent 2017;8(1):41-44.

\section{Source of support: Nil}

Conflict of interest: None

\section{INTRODUCTION}

The residues persistence from the obturation material is a frequent problem in the endodontic retreatment cases. ${ }^{1,2}$ The persistence of these residues can negatively interfere on the sealer adhesion to the root dentin, compromising the success of endodontic treatment and favoring the infection in the root canal. ${ }^{3,4}$

New technological resources have been recommended to optimize the surgical procedures and to facilitate the root canal cleaning. ${ }^{5}$ Despite the rotary instruments facilitate the gutta-percha removal, these instruments do not eliminate the gutta-percha completely. ${ }^{1,6}$ Therefore, rotary instruments associated with organic solvents have been indicated to improve the residues removal. ${ }^{7,8}$

ProTaper retreatment instruments associated with xylol is one of the most effective and rapid techniques to reach working length. ${ }^{9}$ The chloroform is another solvent recommended to be associated with reciprocating, and with the rotary instruments. ${ }^{8}$ However, the solvent effect and residues removal capacity of the root canal of these substances are insignificant. ${ }^{10}$

Endosolv-R is a solvent for phenolic resin-formaldehyde sealers. ${ }^{11}$ Its chemical composition presents formamide and 2-phenylethanol with the former being the major ingredient. ${ }^{12}$ It is more effective in softener epoxy-based sealer, such as AH 26, AH Plus, and Adseal, in relation to xylol. ${ }^{13}$ The residue removal of the epoxybased sealer (AH Plus) from the dentin preventing the adhesion deterioration of the self-etch adhesive systems is another favorable property. ${ }^{12}$

By the other side, the previous obturation techniques and organic solvent used can influence on the persistence of residues in the root canal after endodontic retreatment. ${ }^{14,15}$ This situation occurs in previously obturated canals by single cone tecnhique. ${ }^{16}$ 
So, there is possible relation between the chemical nature of the employed solvent and the retreatment rotary instruments with the root canal obturation technique that was previously performed. However, there are no studies evaluating the effects of xylol or Endosolv-R solvents associated with retreatment rotary instrument in root canals previously obturated by lateral compaction or single cone technique.

Therefore, the aim of this study was to quantify the persistence of residues after the endodontic retreatment in root canal previously filled by lateral compaction or single cone with the use of solvent (xylol or Endosolv-R) associated with ProTaper retreatment system, in the cervical and root apical segments.

\section{MATERIALS AND METHODS}

Forty human uniradicular teeth with single root canal, extracted for periodontal reasons, with similar anatomy, were selected and maintained in $0.1 \%$ thymol solution at $4^{\circ} \mathrm{C}$. The crowns were removed with a diamond disk (KG Sorensen, Barueri, São Paulo, Brazil), leaving 16 mm of radicular length. A \#15K file (Maillefer, Ballaigues, Switzerland) was introduced in the root canal until it was visible at the radicular apex. The working length was established $1.0 \mathrm{~mm}$ shorter than the total radicular length and was confirmed radiographically.

The root canals were prepared by ProTaper Rotary System technique to F2 instrument (Maillefer, Ballaigues, Switzerland) in accordance with manufacturer recommendations. The root canals were irrigated with $5.0 \mathrm{~mL}$ of $2.5 \%$ sodium hypochlorite (Asfer, São Caetano do Sul, São Paulo, Brazil) at each instrument change. After chemicalmechanical preparation, an additional irrigation with $17 \%$ ethylenediaminetetraacetic acid (EDTA) was performed for 1 minute followed by a final flush with $5 \mathrm{~mL}$ of $2.5 \%$ sodium hypochlorite solution.

The root canals were dried using paper points. In sequence, 20 root canals were filled with \#25 gutta-percha master point (Dentsply, Petropolis, Rio de Janeiro, Brazil) by lateral compaction technique, and 20 root canals were filled with F2 ProTaper gutta-percha point (Dentsply, Petropolis, Rio de Janeiro, Brazil) by single cone technique. ${ }^{17}$ A epoxy-based sealer (AH Plus, Dentsply DeTrey $\mathrm{GmbH}$, Konstanz, Germany) was used in all root canals. The coronal radicular access was closed with temporary restorative material (Cavit, 3M, St. Paul, MN, USA). The roots were immersed in artificial saliva that was renewed to each 2 weeks and maintained at $37^{\circ} \mathrm{C}$ for 6 months.

After this period, the roots were embedded in acrylic resin and transversely sectioned $7.5 \mathrm{~mm}$ from the radicular cervical face and two root segments (cervical and apical) were obtained and individually placed in a plastic device. Radiographs were taken from each segment in the buccolingual and mesiodistal direction using digital radiography system.

After the initial radiographic image (R1) was obtained, the teeth were randomly divided into four groups $(n=10)$ and submitted to several endodontic retreatment protocol. In G1 (lateral compaction and xylol), D1, D2, and D3 instruments (ProTaper Universal Retreatment, Dentsply Maillefer, Ballaigues, Switzerland) were used sequentially, in crown-down direction, until the apical working length previously established, as described by Só et al. ${ }^{5} \mathrm{At}$ each instrument change, $0.1 \mathrm{~mL}$ of xylol (Synth, Diadema, São Paulo, Brazil) was introduced in the root canal. In G2 (lateral compaction and Endosolv-R), the sequence was similar to G1, but by using Endosolv-R (Septodont, Saint-Maur-des-Fossés, Cedex, France) as organic solvent. Same procedures were performed in G3 (single cone and xylol) and G4 (single cone and Endosolv-R), but using xylol and Endosolv-R, root canal previously filled by single cone technique.

In sequence, the root canals were irrigated with $5.0 \mathrm{~mL}$ of $2.5 \%$ sodium hypochlorite, aspirated and dried with paper point. A new radiographic (R2) was taken in both directions similar to the first radiograph. The area occupied by the root canal obturation in R1 and the area occupied by residues after the retreatment protocols in

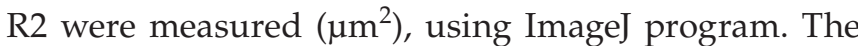
area occupied by residues (R2) was transformed into percentage in relation to the original area occupied by the root canal obturation (R1). The data were statistically analyzed by Kruskal-Wallis test $(\mathrm{p}=0.05)$.

\section{RESULTS}

Table 1 presents the median, maximum, and minimum values in percentage of persistence of residues from the

Table 1: Median, maximum, and minimum values in percentage of persistence of residues from the root canal obturation after different solvents associated with the obturation technique previously performed, in the mesiodistal and buccolingual direction in the cervical segment revealed by radiographic analysis

\begin{tabular}{llll}
\hline & & Median & Min/Max values \\
\hline G1 & MD & 50.11 & $33.33-80.00$ \\
& VL & 36.66 & $20.00-66.66$ \\
G2 & MD & 30.30 & $0.00-62.50$ \\
& VL & 17.30 & $2.00-50.00$ \\
G1 & MD & 41.42 & $0.00-100.00$ \\
& VL & 36.66 & $14.28-75.00$ \\
G1 & MD & 48.07 & $33.33-66.66$ \\
& VL & 20.85 & $6.66-32.53$ \\
\hline
\end{tabular}

*There were no differences among the groups. G1 - lateral compaction and xylol; G2 - lateral compaction and Endosolv-R; G3 - single cone and xylol; and G4 - single cone and Endosolv-R. $\mathrm{MD}$, mesiodistal radiograph; VL, buccolingual radiograph; Min, minimum; Max, maximum 
Table 2: Median, maximum, and minimum values in percentage of persistence of residues from the root canal obturation after different solvents associated with the obturation technique previously performed, in the mesiodistal and buccolingual direction in the apical segment revealed by radiographic analysis

\begin{tabular}{llll}
\hline & & Median & Min/Max values \\
\hline G1 & MD & 50.00 & $10-100$ \\
& VL & 36.50 & $9-82$ \\
G2 & MD & 37.50 & $2-100$ \\
& VL & 26.00 & $2-83$ \\
G1 & MD & 28.77 & $7-100$ \\
& VL & 17.50 & $11-96$ \\
G1 & MD & 18.71 & $1-100$ \\
& VL & 16.50 & $2-45$ \\
\hline
\end{tabular}

${ }^{*}$ There were no differences among the groups. G1 - lateral compaction and xylol; G2 - lateral compaction and Endosolv-R; G3 - single cone and xylol; and G4 - single cone and Endosolv-R. $\mathrm{MD}$, mesiodistal radiograph; VL, buccolingual radiograph; Min, minimum; Max, maximum

root canals obturation after different protocols of endodontic retreatment related to the obturation technique previously performed, showed by radiographic analysis in mesiodistal and buccolingual directions in the cervical segment. Table 2 shows similar results of the apical segment.

There were no significant differences among the groups ( $p>0.05)$, regardless of the analyzed cervical or apical root segment, the radiographic direction, the endodontic retreatment protocol, or the root canal obturation technique previously performed.

\section{DISCUSSION}

Regardless the obturation technique previously performed, the xylol solvents and Endosolv-R presented similar persistence of residues from root canal obturation, regardless of the root segment and radiographic direction.

This study showed that the persistence of residues using Endosolv R was not different from using xylol, regardless of the root canal obturation was previously performed by lateral compaction or by single cone technique. Shenoi et $\mathrm{a}^{13}$ observed that the Endosolv-R presented higher solubilization potential of epoxy-based sealers (AH 26 and AH Plus) than xylol. On the contrary, in the root canal obturation the principal material present is the gutta-percha and the solvent action of these substances on the gutta-percha are similar. ${ }^{12,17}$ Therefore, this fact favored the results from the retreatment protocols were similar.

Intracanal residues persistence is related to the local anatomy. After endodontic retreatment, these residues tend to persist especially in the radicular apical third and in the root canal irregularities..$^{18}$ All root canals used in the present study were previously instrumented and standardized using F2 instrument, facilitating the D3 rotary instrument access until the working length. This phenomenon was also contributed by the fact that the gutta-percha to be the main filling material. ${ }^{17-19}$

As the root canals were previously standardized using F2 instrument, a root canal circular shape was obtained favoring the rotary instruments action. ${ }^{18,19}$ The persistence of gutta-percha residues and/or endodontic sealer tends to be similar in the buccolingual and mesiodistal direction once the root canal anatomy is circular. ${ }^{20}$

Therefore, regardless of root canal obturation technique previously performed, the solvents associated with rotary retreatment instruments were ineffective for the complete removal of root canal obturation. Further studies should be conducted to assess the effects of the complementation of the chemical-mechanical to removal residues of root canal filling after endodontic retreatment.

\section{CONCLUSION}

The persistence of residues from the root canal obturation was similar after endodontic retreatment using retreatment ProTaper system associated with xylol or Endosolv-R, in lateral compaction and single cone techniques, regardless of the root segment analyzed.

\section{CLINICAL SIGNIFICANCE}

The persistence of residues in the root canal compromises the success of the endodontic treatment. Only rotary retreatment instruments and solvents (xylol or Endosolv-R) were ineffective for the root canal obturation removal performed by lateral compaction or single cone technique.

\section{REFERENCES}

1. Guiotti FA, Kuga MC, de Toledo Leonardo R, ChávezAndrade GM, Magro MG, Cavenago BC, Faria G. Effectiveness of ProTaper retreatment system associated with organic solvents in the removal of root canal filling material. World J Dent 2013 Jul;4(3):203-207.

2. Duarte MA, Só MV, Cimadon VB, Zucatto C, Vier-Pelisser $\mathrm{FV}$, Kuga MC. Effectiveness of rotary or manual techniques for removing a 6-year-old filling material. Braz Dent J 2010;21(2):148-152.

3. Rached-JuniorFJ,Sousa-NetoMD,Souza-Gabriel AE,DuarteMA, Silva-Sousa YT. Impact of remaining zinc oxide-eugenolbased sealer on the bond strength of a resinous sealer to dentine after root canal retreatment. Int Endod J 2014 May;47(5):463-469.

4. Chandra A. Discuss the factors that affect the outcome of endodontic treatment. Aust Endod J 2009 Aug;35(2):98-107.

5. Só MV, De Figueiredo JA, Freitas Fachin EV, Húngaro Duarte MA, Pereira JR, Kuga MC, Da Rosa RA. Clinical microscopic analysis of ProTaper retreatment system efficacy considering root canal thirds using three endodontic sealers. Microsc Res Tech 2012 Sep;75(9):1233-1236. 
6. Silva EJ, Orlowsky NB, Herrera DR, Machado R, Krebs RL, Coutinho-Filho Tde $\mathrm{S}$. Effectiveness of rotatory and reciprocating movements in root canal filling material removal. Braz Oral Res 2015 Dec;29:1-6.

7. Sa lam BC, Koçak MM, Türker SA, Koçak S. Efficacy of different solvents in removing gutta-percha from curved root canals: a micro-computed tomography study. Aust Endod J 2014 Aug;40(2):76-80.

8. Zuolo AS, Mello JE Jr, Cunha RS, Zuolo ML, Bueno CE. Efficacy of reciprocating and rotary techniques for removing filling material during root canal retreatment. Int Endod J 2013 Oct;46(10):947-953.

9. Reddy N, Admala SR, Dinapadu S, Pasari S, Reddy MP, Rao MS. Comparative analysis of efficacy and cleaning ability of hand and rotary devices for gutta-percha removal in root canal retreatment: an in vitro study. J Contemp Dent Pract 2013 Jul;14(4):635-643.

10. Kuga MC, Faria G, Rossi MA, do Carmo Monteiro JC, Bonetti-Filho I, Berbert FL, Keine KC, Só MV. Persistence of epoxy-based sealer residues in dentin treated with different chemical removal protocols. Scanning 2013 Jan-Feb;35(1): 17-21.

11. Gambrel MG, Hartwell GR, Moon PC, Cardon JW. The effect of endodontic solutions on resorcinol-formalin paste in teeth. J Endod 2005 Jan;31(1):25-29.

12. Roberts S, Kim JR, Gu LS, Kim YK, Mitchell QM, Pashley DH, Tay FR. The efficacy of different sealer removal protocols on bonding of self-etching adhesives to $\mathrm{AH}$ plus-contaminated dentin. J Endod 2009 Apr;35(4):563-567.

13. Shenoi PR, Badole GP, Khode RT. Evaluation of softening ability of Xylene and Endosolv-R on three different epoxy resin based sealers within 1 to 2 minutes - an in vitro study. Restor Dent Endod 2014 Feb;39(1):17-23.

14. Ma J, Al-Ashaw AJ, Shen Y, Gao Y, Yang Y, Zhang C, Haapasalo M. Efficacy of ProTaper universal rotary retreatment system for gutta-percha removal from oval root canals: a micro-computed tomography study. J Endod 2012 Nov;38(11): 1516-1520.

15. Zuolo ML, Imura N, Ferreira MO. Endodontic retreatment of thermafil or lateral condensation obturations in post space prepared teeth. J Endod 1994 Jan;20(1):9-12.

16. Hess D, Solomon E, Spears R, He J. Retreatability of a bioceramic root canal sealing material. J Endod 2011 Nov;37(11): 1547-1549.

17. Rodrigues A, Bonetti-Filho I, Faria G, Andolfatto C, Camargo Vilella Berbert FL, Kuga MC. Percentage of gutta-percha in mesial canals of mandibular molars obturated by lateral compaction or single cone techniques. Microsc Res Tech 2012 Sep;75(9):1229-1232.

18. Keleş A, Arslan H, Kamalak A, Akçay M, Sousa-Neto MD, Versiani MA. Removal of filling materials from oval-shaped canals using laser irradiation: a micro-computed tomographic study. J Endod 2015 Feb;41(2):219-224.

19. Takahashi CM, Cunha RS, de Martin AS, Fontana CE, Silveira $\mathrm{CF}$, da Silveira Bueno CE. In vitro evaluation of the effectiveness of ProTaper universal rotary retreatment system for gutta-percha removal with or without a solvent. J Endod 2009 Nov;35(11):1580-1583.

20. Nica L, Grigorie M, Rusu D, Anghel MM, Didilescu A, Stratul SI. Computer-assisted photomicrographic evaluation of root canal morphology after removal of the filling material during retreatment. Rom J Morphol Embryol 2011;52 (suppl 1):443-448. 